\title{
Effekte der aus Schulleitersicht wahrgenommenen Belastungen des schulischen Lernens auf die Unterrichtsgestaltung und die Schülerkompetenzen in Mathematik sowie auf die Schulzufriedenheit
}

\author{
Christoph Helm • David Kemethofer • Robert Moosbrugger • \\ Sandra Bröderbauer $\cdot$ Susanne Luthe
}

Angenommen: 30. August 2016 / Online publiziert: 24. Oktober 2016

(C) Der/die Autor(en) 2016. Dieser Artikel ist eine Open-Access-Publikation.

Zusammenfassung Der vorliegende Beitrag beschäftigt sich mit den Merkmalen der Lernumgebung aus Schulleiterperspektive. Im Zentrum der Analysen steht die Frage, inwiefern aus Schulleitersicht wahrgenommene, das schulische Lernen potentiell beeinträchtigende Faktoren am eigenen Schulstandort im direkten und indirekten Zusammenhang mit den Mathematikleistungen und der Schulzufriedenheit stehen. Bezüglich des indirekten Zusammenhangs gehen wir den Effekten der Belastungsfaktoren auf die aus Schülersicht wahrgenommene Unterrichtsgestaltung nach, welche einen Mediator zwischen den schulstandortspezifischen Rahmenbedingungen und den Schülerleistungen bzw. der Schulzufriedenheit darstellt. Die postulierten Wirkmechanismen wurden auf Basis der Daten der Vollerhebung der österreichischen Bildungsstandardüberprüfung in Mathematik auf der 8. Schulstufe (2012) mittels Mehrebenenstrukturgleichungsmodellierung überprüft. Die Analysen zeigen, dass die erlebte Lernbelastung im Zusammenhang mit der Schülerschaft

Dr. C. Helm ( $₫) \cdot$ Mag. R. Moosbrugger

Abteilung für Pädagogik und Pädagogische Psychologie, Johannes Kepler Universität Linz,

Altenberger Straße 69, 4040 Linz, Österreich

E-Mail: christoph.helm@jku.at

Mag. R. Moosbrugger

E-Mail: robert.moosbrugger@jku.at

Dr. D. Kemethofer · S. Bröderbauer, M.A. · Dipl.-Psych. S. Luthe

Bundesinstitut für Bildungsforschung, Innovation und Entwicklung des österreichischen

Schulwesens, Alpenstraße 121, 5020 Salzburg, Österreich

Dr. D. Kemethofer

E-Mail: d.kemethofer@bifie.at

S. Bröderbauer, M.A.

E-Mail: s.broederbauer@bifie.at

Dipl.-Psych. S. Luthe

E-Mail: s.luthe@bifie.at 
(mangelnde Deutschkenntnisse, Elternunterstützung, Disziplin) über den Sozialindex hinaus im negativen Zusammenhang mit der Schülerleistung steht. Auch ein erlebter Mangel an Unterstützungspersonal trägt zu niedrigerer Schülerleistung und Schulzufriedenheit bei. Darüber hinaus steht die erlebte Lernbelastung weder direkt noch indirekt über die Unterrichtsgestaltung im Zusammenhang mit den Schüleroutcomes. Die Diskussion der Ergebnisse fokussiert auf den starken Einfluss des Sozialindex auf das schulische Lernen und stellt mögliche Gegenmaßnahmen auf Ebene der Bildungspolitik, der Schule und des Unterrichts vor.

Schlüsselwörter Rahmenbedingungen des Lehrens und Lernens · Schulleiterbefragung · Kontexteffekte

\title{
Effects of obstacles to learning perceived by school leaders on instructional quality and student outcomes in mathematics as well as school satisfaction
}

\begin{abstract}
The present study investigates features of learning environments from an unexplored perspective: the school leader perspective. At the core of our analyses is the question, in how far obstacles to learning perceived by school leaders are directly and indirectly related to student performance in mathematics and to school satisfaction. With regard to the indirect effect we assume that the effect of obstacles to learning on student outcomes is mediated by instructional quality. These hypothesised effects are tested by means of multilevel latent structure equation modeling on the basis of data of the Austrian educational standard testing in mathematics at grade 8 in 2012. The analyses show that the assumed effects are not confirmed by the data. In total, the obstacles to learning perceived by school leaders are neither directly nor indirectly associated with student performance and school satisfaction. However, there are two exceptions: First, obstacles to learning with regard to students (lack of German skills, lack of parental support, lack of discipline) are associated with lower school performance. Second, the perceived lack of support staff in school is negatively related to school performance and satisfaction. The discussion of the results focuses on the strong effect of a school's social index on teaching and student learning. We present potential measures at different levels of the school system to appropriately respond to the challenges that come along with the social influence.
\end{abstract}

Keywords Determinants of learning and instruction - School leader survey · Climate effects

\section{Einleitung}

Schulisches Lernen kann als ein komplexes Zusammenspiel von individuellen Schülermerkmalen und Merkmalen der Lernumgebung beschrieben werden. Der vorliegende Beitrag beschäftigt sich mit den Merkmalen der Lernumgebung, und zwar aus einer bisher kaum erforschten Perspektive: der Schulleiterperspektive. Deren Erfor- 
schung ist hoch relevant, da sie ein zentrales Merkmal für die erfolgreiche Steuerung des Lehr-Lerngeschehens am Schulstandort ist (Bonsen 2010; Huber und Niederhuber 2004). Im Zentrum der Analysen steht die Frage, inwiefern aus Schulleitersicht wahrgenommene, das schulische Lernen potentiell beeinflussende Faktoren am eigenen Schulstandort im Zusammenhang mit Schüleroutputs stehen. Darüber hinaus gehen wir den Effekten der schulstandortspezifischen Einflussfaktoren auf die aus Schülersicht wahrgenommene Unterrichtsgestaltung nach.

Die Untersuchung der schulischen Rahmenbedingungen (z. B. zum Lehrprofessionswissen, zu Effekten der Schulautonomie) für den Lernerfolg von Schüler/inne/n stellt ein aktuelles Anliegen dar. Auch die Untersuchung der Zusammensetzung von Klassen und Lerngruppen wurde unter dem Begriff „Kompositionseffekte“ Gegenstand zahlreicher Untersuchungen (z. B. Biedermann et al. 2015; Scharenberg 2012). Die vorliegende Studie erweitert diese Forschungsbemühungen, indem die bisher vernachlässigte Schulleiterperspektive als Informationsquelle für die das schulische Lernen beeinflussenden Faktoren herangezogen werden. Dafür nutzen wir Daten aus einer Vollerhebung bei österreichischen Schulleitungen und Schüler/inne/n der Sekundarstufe I. Aus pragmatischen - zur Analyse liegen Bildungsstandarddaten vor - und inhaltlichen Gründen - guter Unterricht ist nach Helmke (2009) lernwirksamer Unterricht, was im Fachunterricht insbesondere das fachliche Lernen betrifft - bilden in der vorliegenden Untersuchung leistungsbezogene und sozioemotionale Aspekte die Kriteriumsvariable.

Ausgehend von Dittons (2000) Rahmenmodell der Schulqualität nehmen wir an, dass zentrale, standortspezifische Rahmenbedingungen in Hinblick auf die Lehrpersonen (Wissen und Motivation), die Schülerzusammensetzung und die Ausstattung der Schule im Zusammenhang mit Aspekten der Unterrichtsgestaltung stehen. Diese Aspekte der Lernumgebung sollten wiederum auf die Schüleroutputs im Fach Mathematik wirken. Um diese Annahmen einer empirischen Prüfung zu unterziehen, wurde ein Mehrebenenstrukturgleichungsmodell mit latenten Konstrukten geschätzt.

Entsprechend dem Aufbau des vorliegenden Beitrags wird im folgenden Abschnitt 2 Dittons Modell der Schulqualität in seinen Grundzügen vorgestellt und die dort skizzierte Beziehung zwischen den schulischen Rahmenbedingungen und den Unterrichtsprozessen mit theoriebasierten Überlegungen gefüllt. Abschn. 3 beschreibt das Datenmaterial, die erhobenen Konstrukte und die eingesetzten Analyseverfahren. In Abschn. 4 und 5 werden die Ergebnisse der Hypothesenprüfung vor dem Hintergrund der Implikationen für die Schulpraxis dargestellt sowie die Grenzen der Studie diskutiert.

\section{Theoretisches Rahmenmodell, Forschungsstand und abgeleitete Hypothesen}

\subsection{Theoretisches Rahmenmodell}

Ditton (2000) legt seinem Modell von Schulqualität eine strukturelle und eine dynamische Dimension zugrunde. Mit der strukturellen Dimension ist das Mehrebenensystem Schule beschrieben und damit die Notwendigkeit, Determinanten der 


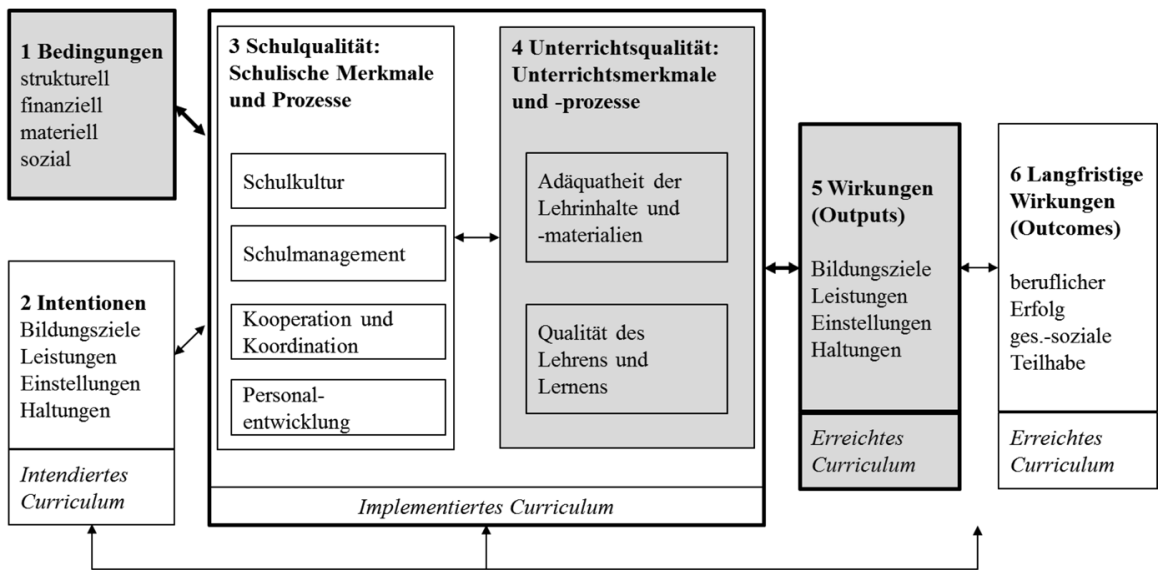

Abb. 1 Qualität in Schulen (Ditton 2000, S. 79)

Erreichung schulischer Ziele auf den verschiedenen Ebenen zu verorten und die wechselseitige Verschränkung der Determinanten über diese Ebenen hinweg zu berücksichtigen. Die dynamische Dimension beschreibt die Idee, dass Inputfaktoren (z. B. strukturelle Bedingungen, Ressourcen) zu schulischen Outputs (Bildungsziele) führen. Eine wesentliche dritte Grundannahme des Modells liegt in der These der primären Bedeutung proximaler Faktoren, d. h., dass Effekte v. a. von Faktoren (z. B. Schülermerkmale) zu erwarten sind, die dem Zielkriterium (z. B. Bildungsziele) nahestehen, während distale Faktoren (z.B. Merkmale der Schule) eher vermittelt über die proximalen Determinanten wirken. Dennoch dürfen die auf Schulebene anzusiedelnden Faktoren nicht ignoriert werden, da die höheren Ebenen als Unterstützungssysteme bzw. Handlungsrahmen der darunterliegenden Ebenen anzusehen sind (vgl. Abb. 1). Ditton (2000, S. 79 f.) hebt deren Bedeutung sogar hervor, indem er die Wechselbeziehungen von Schulbetrieb und Unterricht als ,eigentlichen Kernbereich“ der Schulqualität ansieht.

Ditton (2000, S. 87 f.) geht davon aus, dass schulische Ziele (Block 5 und 6) v. a. dann erreicht werden, wenn Einzelschulen und Lehrer/innen im Einklang mit als verbindlich angesehenen und akzeptierten Zielen (Block 2) und vor dem Hintergrund ihrer ,jeweils spezifischen Bedingungskonstellationen ihrer Schule und in deren Kontext [Block 1, die Autor/inn/en] adaptive Leistungen erbringen." In der vorliegenden Untersuchung fokussieren wir auf genau diesen ersten Block des Modells. Dabei nehmen wir „Bedingungskonstellationen“ in den Blick, die aus Schulleiterperspektive als potentiell hemmend für das Schülerlernen wahrgenommen werden (im Folgenden kurz „Belastungsfaktoren“). Folgende Zusammenhänge stehen im Fokus unserer Analysen (siehe die Hervorhebungen in Abb. 1):

Die aus Schulleiterperspektive wahrgenommenen Belastungsfaktoren am Schulstandort stellen standortspezifische Rahmenbedingungen dar (Block 1), 
die negativ auf Aspekte des Unterrichts (Block 4) wirken, welche wiederum im Zusammenhang mit den Schüleroutputs (Block 5) stehen.

Wir gehen von der Annahme aus, dass eine nachteilige Zusammensetzung von für die Erreichung der Bildungsziele verantwortlichen Akteursgruppen (v. a. Lehrer/ innen und Schüler/innen) sowie Mängel in den zur Leistungserbringung notwendigen Rahmenbedingungen (v. a. Ressourcenausstattung einer Schule) die Unterrichtsgestaltung bzw. das Lehrerhandeln beeinflussen. Da bezüglich dieser Annahmen das Ditton-Modell ein abstraktes Rahmenmodell darstellt, versuchen wir es im Folgenden mit spezifischen Konstrukten und theoriegeleiteten Hypothesen zu zentralen Einflussbereichen der Unterrichtsgestaltung (,Lehrer/innen“, „Schüler/innen“ und „Ressourcenausstattung“") zu füllen.

\subsection{Forschungsstand}

Aspekte der Lehrer/innen: Aktuelle Forschungsbemühungen zum Bereich „Lehrer/innen" nehmen insbesondere deren Kompetenzen in den Fokus (z. B. Baumert und Kunter 2006). Dabei wird nicht nur die Rolle des Lehrerprofessionswissens, sondern auch die der Lehrermotivation im Rahmen der Unterrichtsgestaltung erforscht (z. B. König et al. 2013). Den Grundstein für diesen aktuellen Trend hat Shulman (1986, 1987) mit seinen Arbeiten zur Bedeutung des Lehrerprofessionswissens für die Vermittlung von Konzepten im Unterricht gelegt. Shulman (1987, S. 8) definiert das fachdidaktische Wissen als ,special amalgam of content and pedagogy“, das erfolgreiche Lehrpersonen befähigt, Inhalte verständlich zu vermitteln. In vielen Disziplinen (z. B. Mathematik, Naturwissenschaften, Rechnungswesen) wird diese Form des Wissens über das Lehrerwissen zum a) kognitiven Aktivierungspotenzial von Aufgaben, b) zu den Schülerkognitionen und c) zu verschiedenen Repräsentations- und Erklärungsmöglichkeiten definiert. In mehreren Studien konnte bereits nachgewiesen werden, dass das fachdidaktische Lehrerwissen nicht nur mit höheren Schülerleistungen im Zusammenhang steht, sondern auch mit einer stärker kognitiv aktivierenden Unterrichtsgestaltung einhergeht (z. B. Baumert und Kunter 2011; Darling-Hammond 2000; Hill et al. 2005; Kunter et al. 2013). Es ist naheliegend, dass Lehrpersonen, die bspw. über ein höheres Wissen zu typischen Schülerfehlvorstellungen verfügen, auch eher im Stande sind, Verstehensfragen an Schüler/innen zu stellen oder unterschiedliche Lösungswege zu diskutieren (bspw. Kunter et al. 2013). Das Lehrerprofessionswissen wirkt aber nicht nur auf das Unterrichtsqualitätsmerkmal der kognitiven Aktivierung, sondern auch auf andere Bereiche wie die Individualisierung im Unterricht (Baumert et al. 2010; Kunter et al. 2013). Es konnte gezeigt werden, dass ein höheres Professionswissen auch mit einer höheren Lernerunterstützung einhergeht.

Die Befunde zeigen darüber hinaus, dass auch motivationale Aspekte der Lehrkraft auf die Unterrichtsgestaltung wirken. So beeinflusst in der Studie von Kunter et al. (2013) der Lehrenthusiasmus das lernförderliche Ausmaß der Lehrer-Schüler-Beziehung sowie das Classroom Management. Auch Studien zur Selbstbestimmungstheorie belegen diesen Zusammenhang zwischen Lehrermotivation und Unterrichtsgestaltung (für einen Überblick siehe Kunter (2011)). Die Selbstbestimmungs- 
theorie von Deci und Ryan (1993) geht davon aus, dass intrinsische Motivation aus wahrgenommener Autonomie, Kompetenzerleben und sozialer Eingebundenheit hervorgeht. In den Studien von Müller et al. (2008), Pelletier et al. (2002) sowie Roth et al. (2007) konnte nachgewiesen werden, dass zwischen der intrinsischen Motivation der Lehrpersonen und ihrem autonomieunterstützendem Lehrerverhalten positive Zusammenhänge bestehen.

Neben diesen Lehrermerkmalen analysieren wir auch die Rolle des Unterstützungspersonals, das ein Charakteristikum der Neuen Mittelschule in Österreich darstellt, für die Unterrichtsgestaltung. Bisher wurde diese Rolle, mit wenigen Ausnahmen, kaum erforscht: Alborz et al. (2009) fassen die Befunde aus dem angloamerikanischen Raum zusammen und zeigen, dass in Klassen mit Unterstützungspersonal häufiger kreative, anwendungsorientierte und kooperative Lernformen in Kleingruppen sowie individualisiertes Lernen zum Einsatz kommen. Für Österreich konnten Helm et al. (2015) positive Effekte des Teamteaching auf die Unterrichtsgestaltung (stärkere Schülerorientierung, mehr Freiraum zum selbständigen Lernen, stärker fördernder Unterricht) nachweisen. Ein Mangel an notwendigem Unterstützungspersonal (z. B. Sozialarbeiter/innen) könnte laut Schmich (2010) dagegen dazu führen, dass Lehrpersonen zum Teil Aufgaben übernehmen, für die sie nicht ausgebildet sind und die auch nicht in ihrem Aufgabenbereich liegen (z. B. die ,psychologische“ Betreuung von Schüler/inne/n).

Aspekte der Schüler/innen: Zahlreiche wissenschaftliche Studien verweisen darauf, dass die Schülerzusammensetzung einer Klasse und das Lehrerverhalten bzw. die Unterrichtsgestaltung in Wechselwirkung zueinander stehen.

Studien zur Wirkung der Lernumgebung auf bestimmte Schülergruppen: Die Befunde der ,Aptitude-Treatment-Interaction“-Forschung (ATI) aus dem angloamerikanischen Raum zeigen, dass Schüler/innen aus benachteiligten sozialen Schichten eher von direkten Unterrichtsformen, leistungsstarke Schüler/innen jedoch eher von offenen Unterrichtsmustern profitieren (vgl. Helmke und Weinert 1997), wobei je nach Kontext differentielle Effekte zu erwarten sind: So zeigt Palardy (2008) auf Basis US-amerikanischer Large Scale-Daten, dass das Schülerlernen in Schulen mit ungünstiger Zusammensetzung der Schülerschaft stärker von schulischen Kontextfaktoren und dem Lehrerhandeln beeinflusst wird, als in Schulen mit günstiger Zusammensetzung. Auch in deutschsprachigen Studien wurde in jüngerer Zeit der Frage nachgegangen, wie Unterricht in heterogenen Klassen aussehen kann. Decristan et al. (2016) kommen zu dem Befund, dass neben Individualisierung vor allem kognitive Aktivierung in heterogenen Klassen zu hohen Leistungen führt. Darüber hinaus konnten Fauth et al. (2016) differentielle Effekte für Schüler/innen mit Migrationshintergrund belegen: Diese Schülergruppe profitiert insbesondere von einem guten Classroom Management, einer konstruktiven Unterstützung und einem positiven Unterrichtsklima.

Studien zur Wirkung der Zusammensetzung bestimmter Schülergruppen auf das Lehrerverhalten: Scharenberg et al. (2015, S. 105) argumentieren in Bezug auf die Klassenkomposition, dass Heterogenität den Lehrkräften ein stärkeres Unterstützungsverhalten, mehr Individualisierung und Engagement abverlangt. Eine solche individualisierende Lehrer-Schüler-Beziehung hängt laut TALIS-Studie 2013 u. a. 
von der Arbeitszufriedenheit von Lehrpersonen ab, die (in 29 Ländern) wiederum von der Klassenkomposition (in Hinblick auf den Anteil an Schüler/inne/n mit Verhaltensproblemen) beeinflusst ist (OECD 2014). Eine der wenigen Studien, die im Längsschnitt Schulkompositionseffekten auf die Lernumgebung nachgeht, ist jene von Opdenakker und van Damme (2001). Auf Basis von Daten aus 52 Schulen und 276 Schulklassen in Flandern wird gezeigt, dass der Anteil an leistungsfähigen und sozial besser gestellten Schüler/innen an einer Schule positiv mit der Lehrerkooperation und der Struktur und Ordnung der Lernumgebungen zusammenhängt. Die Leistungsheterogenität an einer Schule korreliert zudem positiv mit dem Fokus auf Disziplin und Lernstofforientierung. In einer Studie aus Deutschland konnten Rjosk et al. (2014) ebenfalls Kompositionseffekte auf das Lehrerverhalten feststellen: In Klassen mit höherem sozioökonomischen Status (SES) berichteten Schüler/ innen einen stärkeren Fokus auf den Sprachenerwerb. In Klassen mit einem höheren Anteil an Schüler/innen mit Deutsch als Erstsprache wurde zusätzlich häufiger von schülerorientiertem Klima und höherer Struktur bzw. besserer Klassenführung im Unterricht berichtet. Allerdings fielen die Effektstärken gering aus. Eine in diesem Zusammenhang interessante Studie, die die Ursachen des Lehrerverhaltens analysiert, stammt von Agirdag et al. (2013). Die Autoren zeigen, dass die Erwartungshaltung von Lehrperson in Schulen mit einem hohen Anteil an Schüler/inne/n mit Migrationshintergrund und aus sozial schwächeren Familien niedriger ist, was wiederum über geringere Schülerselbstwerte negativ auf die Schülerleistungen wirkt. Ein Studie, die zwar nicht das Lehrverhalten in den Blick nimmt, aber wichtige Aspekte der Lernumgebung, führten Liu et al. (2015) durch. Sie zeigen auf Basis von PISA 2003-Daten aus 28 Ländern, dass das Schulklima (Disziplin) und das positive Schülerverhalten sowie die Arbeitsmoral der Schüler/innen negative Schulkompositionseffekte auf die Schülerleistung erklären können.

Aspekte der Ressourcenausstattung: Die Ausstattung der Schule bildet dann eine relevante Determinante des Lehrerverhaltens, wenn der Mangel an Ressourcen dazu führt, dass bestimmte Unterrichtsmethoden nicht umgesetzt werden können (z. B. Schmich 2010). Beispielsweise kann die Umsetzung schülerzentrierter Unterrichtsformen schnell scheitern, wenn die notwendigen räumlichen Voraussetzungen (z. B. eigene Lernräume, -nischen) nicht gegeben sind. Auch die Individualisierung stößt an ihre Grenzen, wenn Lehrpersonal oder individualisiertes Unterrichtsmaterial fehlt (vgl. Moosbrugger et al. 2016). Darüber hinaus können zu hohe Belastungen durch administrative Tätigkeiten wichtige Ressourcen von Schulleitungen und Lehrkräften für nicht-pädagogische Tätigkeiten (z. B. Maßnahmen der Schulentwicklung, Unterrichtsvor- und -nachbereitung) fehlen (Schmich 2010).

\subsubsection{Forschungsstand zum Belastungserleben von Schulleiter/inne/n}

Bisher wurden die Theorieannahmen lediglich aus der Lehr-Lernforschung, insbesondere der Lehrerprofessions- und der Unterrichtsforschung, abgeleitet. In der Schulleiterforschung gibt es erstaunlich wenige Studien, die die Schulleitung als Informationsquelle heranziehen. Häufig werden dagegen andere Schwerpunkte gesetzt: Sie beschäftigen sich mit der Wirkkraft von Schulleitung (zusammenfassend 
Pietsch 2014), deren Rolle im Rahmen neuer, outputorientierter Steuerung (z. B. Brauckmann und Schwarz 2015), daraus resultierenden Anforderungen und damit verbundenem Leitungshandeln (z. B. Brauckmann und Schwarz 2014; Warwas 2015) bzw. dem eigenen Belastungserleben (für eine Übersicht siehe Warwas (2011)). Allerdings sind für die vorliegende Untersuchung insbesondere die Befunde zum Umgang mit erweiterter schulischer Eigenverantwortung interessant. Sie zeigen, dass die an Schulleitung gestellten Ansprüche unter den bestehenden Regelungen und Arbeitsbedingungen von Schulleitungen nicht hinreichend realisiert werden können. Diese Diskrepanz zwischen Erwartetem und Machbarem verweist auf einen Forschungsbedarf hinsichtlich der Kontextbedingungen der Arbeit an Schulen (Brauckmann und Schwarz 2014). Dazu will vorliegender Artikel einen Beitrag leisten.

\subsection{Abgeleitete Hypothesen}

Vor dem Hintergrund dieser theoretischen Überlegungen und Forschungsbefunde lässt sich das abstrakte Rahmenmodell von Ditton nun mit Blick auf Block 1 und 4 durch folgende Hypothesen konkretisieren:

Hypothese 1: Die aus Schulleiterperspektive wahrgenommenen Belastungsfaktoren in Hinblick auf (1) die Lehrperson (mangelndes Fachwissen und mangelnde Motivation), (2) die ungünstige Schülerzusammensetzung und (3) die ungünstige Ressourcenausstattung an der Schule stehen im negativen Zusammenhang mit a) der schülerzentrierten Unterrichtsgestaltung sowie b) den Schüleroutputs (Schülerleistung und Schulzufriedenheit) in Mathematik.

Hypothese 2: Sowohl die Schüleroutputs als auch die Unterrichtsqualität stehen im Zusammenhang mit der tatsächlichen Schülerzusammensetzung (Sozialindex einer Schule und Schultyp).

Hypothese 3: Mit Blick auf die Beziehung zwischen Unterrichtsqualität und Schüleroutput nehmen wir einen sogenannten Climate-Effekt (Lüdtke et al. 2009) an, d. h. über die individuelle Schülerwahrnehmung hinaus beeinflusst die im Mittel kollektiv wahrgenommene Unterrichtsqualität die Schüleroutputs auf Schulebene.

\section{Methode}

\subsection{Beschreibung des Datenmaterials}

Zur Analyse der aufgeworfenen Fragestellung nutzen wir Daten der österreichischen Bildungsstandardüberprüfung 2012 im Fach Mathematik. An der flächendeckend durchgeführten Überprüfung nahmen alle öffentlichen und privaten Schulen mit gesetzlich geregelten Schulartbezeichnungen mit Schüler/inne/n auf der 8. Schulstufe teil. Datengrundlage der vorliegenden Studie ist eine Vollerhebung österreichischer Schüler/innen ( $n=79.678$, $49 \%$ weiblich) an 1416 Schulen, davon 1145 allgemeinbildende Pflichtschulen (APS, $n=53.373$ Schüler/innen) und 271 Allgemeinbilden- 
Tab. 1 Skalenbeschreibung

\begin{tabular}{|c|c|c|c|c|c|c|c|c|}
\hline Faktoren & \# & Beispielitem & $\alpha$ & $\mathrm{ICC} 1 / 2$ & M & SD & Min & $\operatorname{Max}$ \\
\hline $\begin{array}{l}\text { Aspekte der } \\
\text { Unterrichts- } \\
\text { qualität }\end{array}$ & 14 & $\begin{array}{l}\text { Die Lehrperson erklärt } \\
\text { etwas so lange, bis es alle } \\
\text { verstanden haben }\end{array}$ & 0,81 & $0,16 / 0,87$ & 2,53 & 0,55 & 1 & 4 \\
\hline $\begin{array}{l}\text { Belastung: } \\
\text { Lehrer/innen }\end{array}$ & 2 & $\begin{array}{l}\text {... durch einen Mangel an } \\
\text { geprüften Lehrkräften in } \\
\text { Mathematik }\end{array}$ & 0,32 & - & 1,21 & 0,34 & 1 & 4 \\
\hline $\begin{array}{l}\text { Belastung: } \\
\text { Schüler/innen }\end{array}$ & 3 & $\begin{array}{l}\text {... durch mangelnde } \\
\text { Kenntnisse der Schüler/ } \\
\text { innen in der Unterrichtss- } \\
\text { prache Deutsch }\end{array}$ & 0,72 & - & 2,05 & 0,62 & 1 & 4 \\
\hline $\begin{array}{l}\text { Belastung: } \\
\text { Ausstattung }\end{array}$ & 3 & $\begin{array}{l}\text {... durch fehlendes oder } \\
\text { unzulängliches Unter- } \\
\text { richtsmaterial }\end{array}$ & 0,78 & - & 1,50 & 0,63 & 1 & 4 \\
\hline \multicolumn{2}{|c|}{ Schulzufriedenheitl } & $\begin{array}{l}\text { Wie gerne gehst du in die } \\
\text { Schule? }\end{array}$ & - & $0,04 /-$ & 2,36 & 1,05 & 1 & 5 \\
\hline
\end{tabular}

Die konkrete Fragestellung sowie die Antwortkategorien können in BIFIE (2012a, 2012b) nachgelesen werden. Bezüglich der Antwortformate gilt für den Unterrichtsqualitätsfaktor: $1=$ in jeder Stunde; $2=$ in den meisten Stunden; 3 = in einigen Stunden; 4 = nie oder fast nie. Für die Belastungsfaktoren gilt: 1 = ja, sehr stark; 2 = ja, deutlich; 3 = ja, ein wenig; 4 = nein, gar nicht. Für die Schulzufriedenheit gilt: $1=$ sehr gern $5=$ sehr ungern. Die Antwortformate aller Skalen wurden so umkodiert, dass die höchste Ausprägung die höchste Zustimmung darstellt. Der ICC (2) wurde nach Lüdtke et al. (2009) wie folgt berechnet: Interklassenvarianz/(Interklassenvarianz + (Intraklassenvarianz/mittlere Klassengröße))

de Höhere Schulen (AHS, $n=26.305$ Schüler/innen) (Schreiner und Breit 2012). Die beiden Schultypen unterscheiden sich bspw. hinsichtlich der sozialen Komposition der Schülerschaft (die auch mit der Schülerleistung zusammenhängt) und unterschiedlicher Ausbildung der Lehrpersonen. In diesem Aufsatz greifen wir auf Daten aus den Schülerüberprüfungen und auf Informationen des Schulleiter- und des Schülerkontextfragebogens zurück. Mittels der Kontextfragebögen wurde u. a. die Wahrnehmung des Mathematikunterrichts aus Schülersicht erfasst. Tab. 1 enthält testtheoretische Informationen sowie Beispielitems zu den erfassten Konstrukten, die im Folgenden inhaltlich beschrieben werden. Die Skalen wurden auf Basis der in den Kontextfragebögen (Schulleiterberfragung: BIFIE 2012a; Schülerbefragung: BIFIE 2012b) enthaltenen Items gebildet.

\subsubsection{Lernhemmende Faktoren aus Schulleitersicht}

Die aus Schulleitersicht wahrgenommen lernhemmenden Faktoren am Schulstandort wurden wie folgt erfragt: (1) ,Wird das Lernen im Allgemeinen an Ihrer Schule aus Ihrer Sicht durch die folgenden Faktoren beeinträchtigt?" und (2), da es sich um die Standardüberprüfung in Mathematik handelte: „Wird das MATHEMATIK-Lernen an Ihrer Schule aus Ihrer Sicht durch die folgenden Faktoren beeinträchtigt?“ Die lernhemmenden Aspekte sind in Tab. 2 entsprechend ihrer Häufigkeit, in der sie von Schulleitungen als belastend erlebt werden, absteigend sortiert. Die zehn Items lassen sich auf die drei Faktoren „Belastung: Lehrer/innen“, „Belastung: Schüler/innen“, „Belastung: Ausstattung“ reduzieren, wobei die Items „Beeinträchtigung pädagogischer Arbeit durch hohen administrativen Aufwand“ und „Beeinträchtigung durch 
Tab. 2 Belastungsfaktoren des schulischen Lernens am eigenen Schulstandort

\begin{tabular}{ll}
\hline Rahmenbedingungen $(N=1396)$ & Ja (in \%) \\
\hline Allgemein & 61,3 \\
Administrativer Aufwand & 43,5 \\
Fehlende Unterstützung der Schüler/innen durch ihre & \\
Eltern & \\
Mathematik & 23,1 \\
Fehlende Disziplin der Schüler/innen & 22,0 \\
Mangel an Unterstützungspersonal & 15,2 \\
Mangelnde Deutschkenntnisse der Schüler/innen & 14,4 \\
Fehlende oder unzulängliche Computerausstattung & 12,2 \\
Fehlende oder unzulängliche Ausstattung & 5,8 \\
Fehlendes oder unzulängliches Unterrichtsmaterial & 1,7 \\
Mangelnde(s) Engagement/Motivation der Lehrkräf- & \\
te & 1,6 \\
Mangel an geprüften Lehrkräften &
\end{tabular}

Die konkrete Fragestellung sowie die Antwortkategorien können in BIFIE (2012a, 2012b) nachgelesen werden. Skala: $1=$ ja, sehr stark; 2 = ja, deutlich; 3 = ja, ein wenig; 4 = nein, gar nicht. Die Kategorie 1 und 2 wurden zusammengefasst.

einen Mangel an unterstützendem Personal" auf keinem der drei Faktoren laden. Sie wurden als manifeste Prädiktoren im Modell berücksichtigt. Der Faktor „Belastung: Lehrer/innen“" setzt sich aus den beiden Items zum Mangel an geprüften Lehrkräften in Mathematik und zum mangelnden Engagement bzw. zur fehlenden Motivation der Lehrkräfte zusammen. Der zweite Faktor „Belastung: Schüler/innen“ enthält drei Items, die die Deutschkenntnisse und die Disziplin sowie die elterliche Unterstützung der Schüler/innen betreffen. Der letzte Faktor „Belastung: Ausstattung“ bezieht sich auf die unzulängliche Ausstattung in Hinblick auf die Klassenräume bzw. das Schulgebäude, das Unterrichtsmaterial und die Computerausstattung.

\subsubsection{Aspekte der schülerzentrierten Unterrichtsgestaltung}

Der Schülerkontextfragebogen der Standardüberprüfung enthält 14 unterrichtsbezogene Items, die zu einem Faktor zusammengefasst wurden. Dieser kann als globale Schülereinschätzung zur Unterrichtsqualität angesehen werden. Wir sprechen hier absichtlich von Unterrichtsqualität, da die Items auf Aspekte der schülerzentrierten Unterrichtsgestaltung abzielen, die aus theoretischer Perspektive als lernförderlich angenommen werden können (bspw. Boekaerts 1999). So wird etwa nach der Explikationen und Begründung von Lehrzielen durch die Lehrperson gefragt; oder nach der Individualisierung durch den Einsatz von adaptiven Lernaufgaben sowie der Reflexion im Lernprozess etc.

\subsubsection{Schüleroutcomes}

Im Rahmen der Bildungsstandardüberprüfung für Mathematik sollten die Schüler/innen jeweils 48 Aufgaben in einem Zeitraum von 90 min bearbeiten. Mittels 
Rasch-Modellierung wurden die Testaufgaben zu Vergleichszwecken skaliert (Kuhn und Kiefer 2013). Im Durchschnitt erzielten die Schüler/innen eine Leistung von 535 Punkten, bei einer Standardabweichung von 94 Punkten. Die AHS-Schüler/ innen erreichten im Durchschnitt 96 Punkte mehr als APS-Schüler/innen. Dies entspricht rund neun bis zehn mehr gelösten Aufgaben im Test. Informationen zu den erfassten mathematischen Inhaltsbereichen, den zugrunde liegenden Kompetenzmodellen sowie der Testdurchführung können bei Schreiner und Breit (2012) nachgelesen werden.

\subsubsection{Index der sozialen Benachteiligung einer Schule}

Der Index sozialer Benachteiligung einer Schule (im Folgenden kurz „Sozialindex“) setzt sich aus den folgenden vier Merkmalen zusammen: ,i) Anteil der Kinder aus dem unteren Quintil der Sozialstruktur, ii) Anteil der Kinder von Eltern mit maximal Pflichtschulabschluss, iii) Anteil der Kinder mit Migrationshintergrund, und iv) Anteil der Kinder mit nichtdeutscher Alltagssprache“ (Bruneforth et al. 2012, S. 198). Je höher der Wert, desto größer ist die soziale Benachteiligung einer Schule, wobei das absolute Minimum bei einem Wert von 100 liegt. Für unsere Daten liegt der Sozialindex der Schulen im Mittel bei 119 (SD = 13,64). Die Verteilung präsentiert sich als stark linkssteil, d.h., dass zum Zeitpunkt der Datenerhebung nur wenige Schulen eine hohe bzw. sehr hohe Belastung aufwiesen. Darüber hinaus zeigen sich deutliche, schultypenspezifische Unterschiede. So ist eine mittlere bis hohe soziale Belastung in allgemeinbildenden höheren Schulen nur in Ausnahmefällen vorhanden $(7,9 \%)$, während im allgemeinen Pflichtschulbereich mehr als $50 \%$ der Schulen mittel bis sehr hoch sozial belastet sind (Bruneforth et al. 2012; Kemethofer et al. 2015). Da die Merkmale, die zur Bildung des Sozialindex herangezogen wurden stark mit der kognitiven Leistungsfähigkeit zusammenhängen, nehmen wir diesen Index als Kontrollvariable in die Modelle auf. Ferner ergänzen wir das statistische Modell um den Schultyp als weitere kontrollierende Variable.

\subsection{Statistische Analysen}

Für die eben beschriebenen Konstrukte „Belastungsfaktoren“ und „Aspekte der schülerzentrierten Unterrichtsgestaltung“" wurden Messmodelle (vgl. Tab. 3) mit den entsprechenden Items gebildet. Auf Basis dieser Messmodelle wurde die oben beschrie-

Tab. 3 Messmodelle

\begin{tabular}{llllll}
\hline Faktoren & RMSEA & CFI & TLI & SRMR & Faktorladungen \\
\hline $\begin{array}{l}\text { Aspekte der Unterrichtsgestal- } \\
\text { tung }\end{array}$ & 0,027 & 0,95 & 0,94 & $0,03 / 0,10 / 0,11$ & $0,408-0,974$ \\
$\begin{array}{l}\text { Belastungen (3 Faktorenmo- } \\
\text { dell) }\end{array}$ & 0,010 & 0,97 & 0,96 & n. a./n. a/0,03 & $0,641-0,843$ \\
\hline
\end{tabular}

n. $a$. nicht anwendbar. $N=1416$ Schulklassen bzw. 79.678 Schüler/innen. Die Faktorenladungen beziehen sich im Fall der Unterrichtsgestaltung auf die Lerngruppenebene; im Fall der Belastungen auf die Schulebene. Die drei SRMR-Werte beziehen sich auf die untersuchten Ebenen (Schüler/innen/Klasse/Schule). Da die Belastungsfaktoren nur auf Schulebene modelliert wurden, liegen für die beiden unteren Ebenen keine SRMR-Werte vor 
bene Wirkungskette mittels Mehrebenenstrukturgleichungsmodellierung in Mplus 7 (Muthén und Muthén 1998-2014) getestet. Für die Variable „Aspekte der Unterrichtsqualität“" wurde ein Doubly Latent Model (Marsh et al. 2009) geschätzt, das das Konstrukt durch ein Messmodell auf Schüler- und Schulebene stichprobenund messfehlerbereinigt erfasst. Die Koeffizienten wurden unter Verwendung der Mplus-Option für Maximum Likelihood Schätzer für robuste Standardfehler (MLR) geschätzt. Das heißt, Standardfehler sind gegenüber Verletzungen der Normalverteilungsannahme robust (Yuan und Bentler 2000). Die Normalverteilung ist vor allem bei den Items „Alle Schüler/innen machen die gleichen Übungen“ oder „Ich darf mir aussuchen ob ich alleine, zu zweit oder in einer kleinen Gruppe arbeite“ nicht gegeben, da diese Form des Schülerlernens in der Praxis entweder sehr häufig oder sehr selten vorkommt.

Als Gütekriterien für die Modellanpassung werden der Comparative Fit Index (CFI), der Tucker Lewis Index (TLI) und der Root Mean Square Error of Approximation (RMSEA) sowie der Standardized Root Mean Square Residual (SRMR) herangezogen. Als gute/akzeptable Modellfits werden in der Literatur üblicherweise folgende Werte verwendet (Browne und Cudeck 1993; Hu und Bentler 1999; Little 2013): CFI-Werte $\geq 0,95 / 0,90$, TLI-Werte $\geq 0,95 / 0,90$, RMSEA-Werte $\leq 0,05 / 0,08$ und $\mathrm{SRMR} \leq 0,05 / 0,10$. Die Berücksichtigung der hierarchischen Struktur und die Ermittlung von sogenannten Climate-Effekten nach Lüdtke et al. (2009) erfolgt durch den TYPE = TWOLEVEL Command in Mplus. Dazu wurden die Level 1 (L1) Variablen am Gruppenmittelwert zentriert, um die reinen L1-Effekte zu erhalten, und die Level 2 (L2) Variablen am Gesamtmittelwert zentriert (Lüdtke et al. 2009). Dieses Vorgehen ermöglicht die Untersuchung von Climate-Effekten, nämlich des Zusammenhangs der (1) individuellen sowie (2) kollektiven Schülerwahrnehmung von schülerzentriertem Unterricht mit den Schülerleistungen in Mathematik (Lüdtke et al. 2009). Fehlende Werte wurden durch Multiple Imputation (10 Datensätze) berücksichtigt. Sämtliche Analysen wurden für 10 getrennte Datensätze berechnet. Die Ergebnisse wurden nach Rubin (1987) gepoolt.

\section{Ergebnisse}

\subsection{Befunde zur Hypothese 1 (Belastungsfaktoren an einer Schule)}

In den hier betrachteten Hypothesen spiegelte sich die Frage nach den direkten und indirekten Effekten von am Schulstandort erlebten Belastungsfaktoren des Schülerlernens auf die Outputs wider.

Direkte Effekte: Die Hypothesenprüfung kommt zu dem Ergebnis, das unter Kontrolle des Schultyps und des Sozialindex der Schule lediglich die Belastungsfaktoren „Schüler/innen“ $(\beta=-0,172, p<0,001)$ und ,Mangel an Unterstützungspersonal““ $(\beta=-0,091, p<0,001)$ im negativen Zusammenhang mit der mittleren Schülerleistung einer Schule in Mathematik stehen. Mit Blick auf die Schulzufriedenheit bestätigt sich der wahrgenommene Mangel an Unterstützungspersonal als prädiktiv $(\beta=-0,106, p=0,001)$. 
Indirekte Effekte: Die Befunde zeigen, dass - aufgrund des fehlenden Zusammenhangs der Belastungsfaktoren mit der Unterrichtsqualität - sich auch die Vermutung indirekter Effekte über die Unterrichtsgestaltung auf die Schülerleistungen und Schulzufriedenheit nicht bestätigen lässt. Einzig der Belastungsfaktors „Lehrer/ innen“ steht in einem schwachen, statistisch knapp nicht signifikantem Zusammenhang mit der schülerzentrierten Unterrichtsgestaltung $(\beta=-0,071, p=0,076)$. Die aufgestellten Hypothesen zu den direkten und indirekten Effekten müssen daher mit Ausnahme der berichteten Effekte verworfen werden.

Einschränkend muss allerdings angemerkt werden, dass die hohe Korrelation $(\beta=$ 0,816, $p<0,001)$ zwischen dem Belastungsfaktor "Schüler/innen“ (fehlende Elternunterstützung, mangelnde Deutschkenntnisse, Disziplinprobleme) und dem Sozialindex darauf verweist, dass die beiden Konstrukte ähnliches messen. Daher ist zu erwarten, dass die Effekte des Sozialindex auf den Belastungsfaktor „Schüler/innen“ übergehen, wenn der Sozialindex aus dem Modell genommen wird. Weiterführende Analysen (ohne Tabelle) bestätigen dies.

\subsection{Befunde zur Hypothese 2 (Sozialindex und Schultyp)}

Neben den im Analysefokus stehenden Effekten der Belastungsfaktoren erweisen sich folgende Schulmerkmale als bedeutend für die Vorsage der Unterrichtsgestaltung im Sinne selbstgesteuerten Lernens und der Schüleroutputs.

Unterrichtsgestaltung: Der Sozialindex einer Schule steht im positiven Zusammenhang mit der schülerzentrierten Unterrichtsgestaltung $(\beta=0,330, p<0,001)$, d.h. in sozial belasteten Schulen nehmen Schüler/innen im Durchschnitt deutlich mehr Aspekte eines schülerzentrierten Unterrichts wahr. Darüber hinaus macht der Schultyp einen starken Unterschied, was die schülerzentrierte Unterrichtsgestaltung betrifft $(\beta=0,622, p<0,001)$. In den AHS berichten Schüler/innen über deutlich selteneren Einsatz von schülerzentrierten Lernformen.

Schüleroutputs: Des Weiteren sagen der Sozialindex $(\beta=-0,462, p<0,001)$ und der Schultyp $(\beta=0,536, p<0,001)$ die mittlere Schülerleistung einer Schule vorher. Der Schultypeneffekt spiegelt den Unterschied zwischen APS zu AHS wider, wobei die AHS im Mittel über höhere Schulleistungen verfügt. Beide Effektstärken können als mittel bis hoch bezeichnet werden, da sich die durchschnittliche Schülerleistung um rund eine halbe Standardabweichung (etwa 45-50 Punkte) verändert, wenn sich der Sozialindex einer Schule um eine Standardabweichung verändert bzw. der Schultyp von APS auf AHS wechselt. Interessant ist auch der unerwartet hohe Schultypeneffekt auf die Schulzufriedenheit $(\beta=0,588, p<0,001)$. In den AHS berichten die Schüler/innen über eine höhere Schulzufriedenheit als in den APS (im Schnitt um mehr als eine halbe Standardabweichung).

\subsection{Befunde zur Hypothese 3 (Climate-Effekte)}

Zuletzt möchten wir auf den Climate-Effekt bzgl. der Unterrichtsgestaltung verweisen: Da bei Vorhersage der Mathematikleistung (Abb. 2) die Effekte des schüler- 

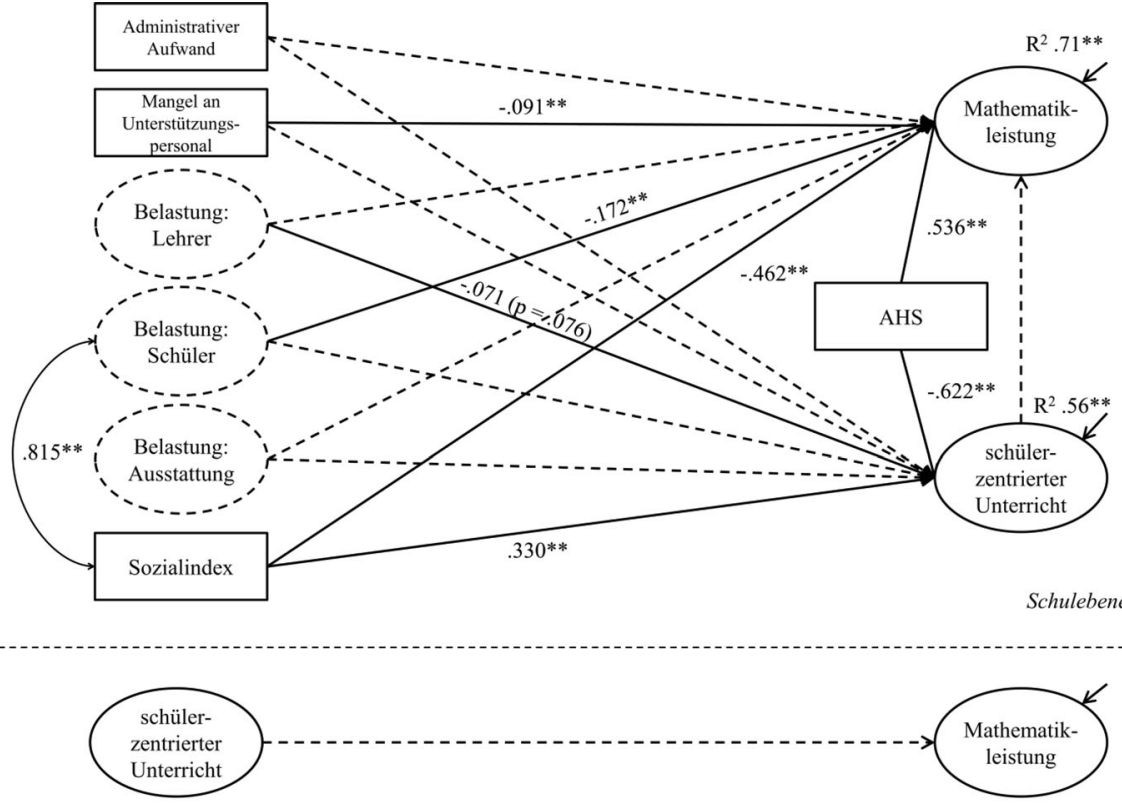

Schülerebene

Schüler/innen $=79678$, Schulen $=1416$ RMSEA: .029, CFI: .92, TLI: .91, SRMR: .037/.091

Abb. 2 Direkte und indirekte Effekte der Belastungsfaktoren auf die Schülerleistungen. Unterbrochene Pfeile stellen geprüfte, aber statistisch nicht signifikante Pfade dar, während die durchgezogenen Pfeile geprüfte und statistisch sowie praktisch signifikante Pfade darstellen. $* p<0,05$, $* * p<0,01$

zentrierten Unterrichts weder auf Schüler- noch auf Schulebene signifikant sind, ergeben sich auch keine Climate-Effekte. Anders verhält es sich im Rahmen der Vorhersage der Schulzufriedenheit (Abb. 3). Hier entfaltet der schülerzentrierte Unterricht über den Level 1-Effekt $(\beta=0,156, p<0,001)$ hinaus auch auf Schulebene einen Climate-Effekt $(\beta=0,330, p<0,001)$ auf die Schulzufriedenheit.

Modellgüte: Die in den Abb. 2 und 3 berichteten Modellfitwerte (Modell „Mathematikleistung", Abb. 2: RMSEA =0,029, CFI 0,92, TLI $=0,91$, SRMR $=$ 0,037/0,091; Modell „Schulzufriedenheit““, Abb. 3: RMSEA = 0,023, CFI = 0,94, TLI $=0,93$, SRMR $=0,031 / 0,088$ ) weisen auf eine akzeptable Abbildung der Modellannahmen durch die empirisch gegebene Datenstruktur hin. In Hinblick auf die Varianzaufklärung der Mathematikleistung und der Unterrichtsgestaltung scheint das Modell auf Schulebene mit den Prädiktoren „Belastung Schüler/innen“, Sozialindex und Schultyp zentrale Determinanten zu beinhalten. Die Varianzaufklärung von $71 \%$ bzw. $56 \%$ auf Schulebene sowie die Fitindizes verweisen auf eine akzeptable Modellgüte. Dies gilt zum Teil auch mit Blick auf die Schulzufriedenheit als Kriteriumsvariable; hier konnten immerhin $22 \%$ der Varianz zwischen den Schulen aufgeklärt werden. 

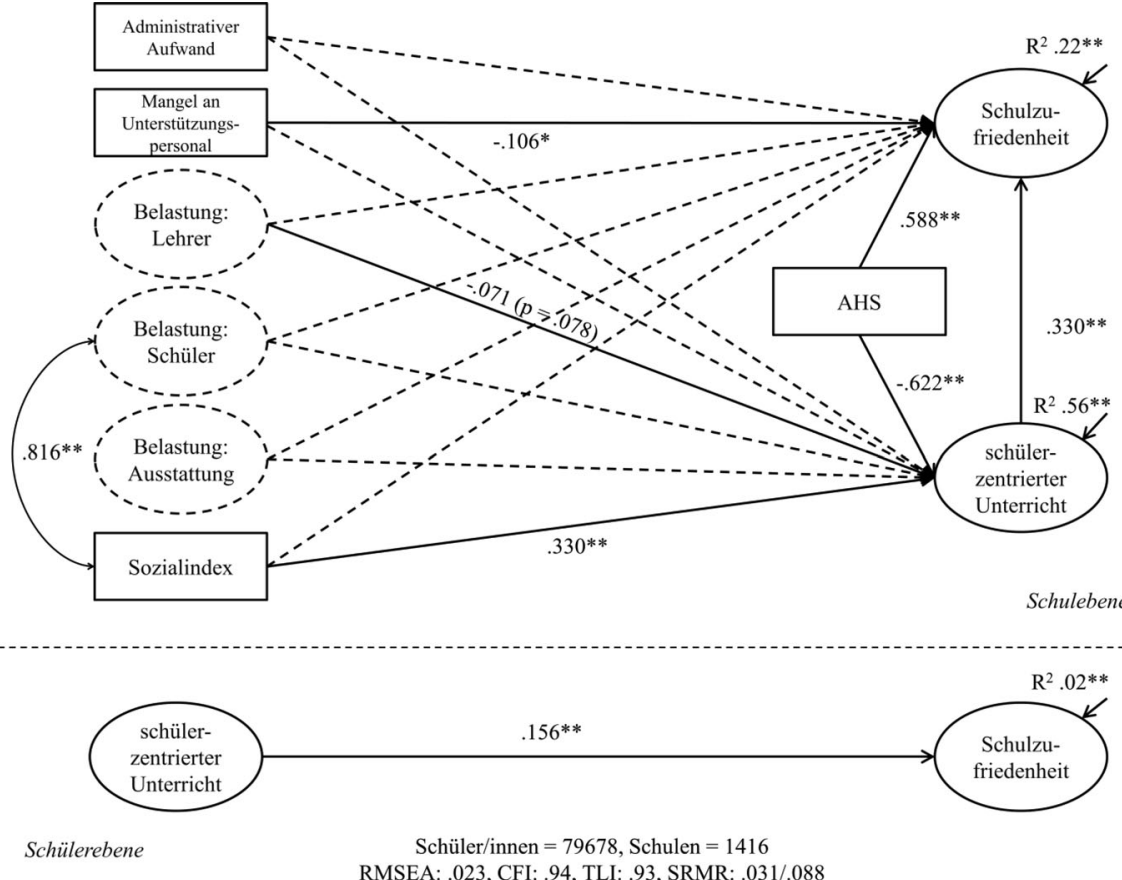

Abb. 3 Direkte und indirekte Effekte der Belastungsfaktoren auf die Schulzufriedenheit. Unterbrochene Pfeile stellen geprüfte, aber statistisch nicht signifikante Pfade dar, während die durchgezogenen Pfeile geprüfte und statistisch sowie praktisch signifikante Pfade darstellen. $* p<0,05$, $* * p<0,01$

\section{Diskussion}

Der vorliegende Beitrag ging der Frage nach, inwiefern die aus Schulleitersicht wahrgenommene, das schulische Lernen potentiell beeinträchtigende Faktoren am eigenen Schulstandort im direkten und indirekten Zusammenhang mit den Mathematikleistungen und der Schulzufriedenheit stehen. Die Hypothese 1, wonach die Belastungsfaktoren in Hinblick auf (1) einen berichteten Mangel an motivierten und fachgeprüften Mathematiklehrpersonen sowie einem Mangel an Unterstützungspersonal, (2) mangelnde Elternunterstützung, Deutschkenntnisse und Disziplin der Schüler/innen sowie (3) mangelnde Ausstattung und einen hohen administrativen Aufwand prädiktiv für die aus Schülersicht berichtete Unterrichtsqualität, die Mathematikleistungen und die Schulzufriedenheit sind, konnte nur teilweise bestätigt werden. Lediglich die im Zusammenhang mit der Schülerklientel erlebten Belastungen korrelieren auf Schulebene bedeutend negativ mit der Mathematikleistung, auch nach Kontrolle des Sozialindex. Es scheint, dass der Faktor „Belastung Schüler/ innen “ über den Sozialindex hinaus relevante, lernhinderliche Information enthält. Vermutlich lässt sich die zusätzliche Varianzaufklärung durch den Mangel an elterlicher Unterstützung und der fehlenden Disziplin der Schüler/innen begründen. Darüber hinaus hängt der erlebte Mangel an Unterstützungspersonal negativ mit der mittleren Leistung und Schulzufriedenheit der Schüler/innen an einer Schule zu- 
sammen. Dieser Befund erscheint plausibel, allerdings können wir nur mutmaßen, worin die tatsächliche Ursache liegt; vermutlich in einer intensiveren und zielgruppengerechteren Betreuung der Schüler/innen durch adäquates Schulpersonal. Hier sind weitere Forschungsbemühungen notwendig. Die wahrscheinlichste Erklärung für das Ausbleiben der vermuteten Effekte der schulischen Rahmenbedingungen liegt womöglich in der distalen Position der Faktoren zu den Schülerleistungen. Auch die Erfassung via Schulleitereinschätzung ist wohl nicht immer reliabel und valide. Zumindest für den Belastungsfaktor „Lehrer/innen“ ist zu erwarten, dass das tatsächliche Niveau der Lehrermotivation und des Lehrerwissen durchaus einen Effekt auf die Unterrichtsgestaltung und die Schülerleistung hat.

Die vorliegenden Befunde zu Hypothese 2 reihen sich in die Befunde der Studien zum starken Einfluss des Sozialindex auf die Schülerleistung (z. B. Bruneforth et al. 2012; Kemethofer et al. 2015) ein (auch wenn die Schulzufriedenheit entgegen unseren intuitiven Erwartungen vom Sozialindex entkoppelt ist). Gleichzeitig ist anzunehmen, dass der Sozialindex und der Schultyp stark mit der kognitiven Leistungsfähigkeit der Schüler/innen konfundiert sind (Helm et al. 2015), sodass die Unterrichtsgestaltung stärker durch die Leistungsfähigkeit als durch die soziale Herkunft beeinflusst ist. Mit Blick auf den positiven Zusammenhang des Sozialindex auf die Unterrichtsgestaltung deckt sich der vorliegende Befund mit den Ergebnissen aktueller ländervergleichenden Analysen von Klieme und Kuger (2016, S. 289). Sie zeigen, dass leistungsschwächere bzw. sozio-ökonomisch schlechter gestellte Schüler/innen von „mehr Unterstützung und (Förder-)Praktiken“ berichten. Auch in der vorliegenden Studie nehmen Schüler/innen aus sozial stärker belasteten Schulen höhere individuelle Förderung wahr. Dieser Befund kann unterschiedlich erklärt werden: Einerseits könnten Lehrpersonen in der Schülerzentrierung den adäquateren und erfolgversprechenderen Unterricht für diese Schülergruppe sehen. Andererseits könnten die Rahmenbedingungen in stark sozial belasteten Schulen und Klassen die Lehrpersonen auch dazu zwingen, stärker schülerzentrierten Unterricht einzusetzen, da traditionelle, lehrerzentrierte Verfahrensweisen dort nicht länger umsetzbar sind (vgl. dazu Helm und Altrichter 2012). Auch zeigt der starke Schultypeneffekt, dass die Unterrichtsgestaltung vermutlich mit dem Bild über die AHS und APS einhergeht. So kann angenommen werden, dass AHS-Lehrpersonen eher einem anderen Bildungsauftrag folgen, bspw. jenem der Elitenbildung, und daher lehrer- und stoffzentrierten, zügigen Unterricht favorisieren. Der Befund, dass in den AHS Schüler/ innen über deutlich selteneren Einsatz von schülerzentrierten Lernformen berichten, ist aus wissenschaftlicher Sicht interessant, da schülerzentrierter Unterricht gerade für diese Schülergruppe Vorteile in Hinblick auf den vertieften Wissenserwerb bringen würde (vgl. die berichteten Befunde der ATI-Forschung).

Aus den vorliegenden Ergebnissen lassen sich Schlussfolgerungen für die Schulund Unterrichtspraxis ableiten. Der starke Zusammenhang des Sozialindex aber auch der wahrgenommenen, fehlenden Elternunterstützung, der mangelnden Deutschkenntnisse sowie der niedrigen Disziplin - mit der Unterrichtsgestaltung und den Schülerleistungen unterstützt auf Ebene der Bildungspolitik die Forderungen nach einer indexbasierten Mittelzuweisung (Bacher et al. 2010). Gemeinsam mit erhöhter Schulautonomie könnte die indexbasierte Mittelzuweisung Schulleitungen genügend Freiraum und Steuerungsmöglichkeiten zur Bewältigung der Heraus- 
forderungen verschaffen (bspw. durch zusätzliche Lehrpersonen für Deutsch als Zweitsprache, Schulpsycholog/inn/en, ...). Die vorliegenden Befunde, insbesondere jene zum Schultyp sowie die in Moosbrugger et al. (2016) berichteten Schulleiteraussagen zur zunehmenden Konkurrenz um leistungsfähige Schüler/innen zwischen AHS und APS, sind darüber hinaus ein weiteres Argument für die Gesamtschule. Für das Schulleiterhandeln bedeuten diese Befunde, dass u. a. durch die Förderung der Kooperation im Lehrerkollegium und der Unterstützung von Maßnahmen für den Umgang mit Heterogenität und leistungsschwachen Klassen förderliche Rahmenbedingungen (z. B. durch adaptive Unterrichtsmodelle) für die Bewältigung der sozialen Belastung geschaffen werden müssen. Auch für die Schulleitungen selbst muss von Seiten der Bildungspolitik das administrative Arbeitspensum reduziert werden, um Freiraum für die eigentlichen Schulleiteraufgaben zu schaffen. Brauckmann und Schwarz (2015) zeigen, dass zentralen Schulentwicklungsaufgaben aufgrund von Überlastungen am Schulstandort nicht nachgekommen werden kann, obwohl diesen Aufgaben von Schulleitungen hohe Bedeutung für die Leitung einer Schule beigemessen wird. Mit Blick auf das Lehrerhandeln zeigen Studien (Decristan et al. 2016; Scharenberg 2012), dass Leistungsheterogenität an sich nicht im Zusammenhang mit der Schülerleistung steht, sondern erst im Zusammenhang mit dem pädagogischen Handeln bedeutsam wird: „Ob Leistungsheterogenität in Klassen produktiv aufgegriffen wird, hängt somit wesentlich von der Unterrichtsgestaltung ab." (Decristan et al. 2016, S. 290) Die Befunde sprechen dafür, dass Anreize geschaffen werden müssen, die besten Lehrpersonen für die sozial am stärksten belasteten Schulen zu gewinnen; eine Forderung, der auch im Nationalen Bildungsbericht 2015 Nachdruck verliehen wird (Biedermann et al. 2015).

Die hier vorgestellten Befunde und Handlungsimplikationen sind vor dem Hintergrund folgender Grenzen der vorliegenden Studie zu interpretieren: Die lernhemmenden Faktoren an einer Schule wurden aus Sicht der Schulleitung erfasst, was explizites Ziel der Studie war und auch den Neuigkeitswert der Studie ausmacht. Auch wenn wir davon ausgehen, dass die Schulleitung aufgrund ihrer zentralen Rolle am Schulstandort den vermutlich besten Einblick in das Schulgeschehen hat, müssten die Aussagen z. B. durch objektive Informationsquellen wie externe Beobachter/ innen validiert werden. So ist in Hinblick auf die Einschätzung der schulischen Ausstattung zu erwarten, dass eher Antworten gegeben werden, die den Wunsch nach mehr Ressourcen verkörpern und weniger den tatsächlichen Zustand widerspiegeln. Zur Schulleiterselbsteinschätzung kommt hinzu, dass die vorgelegten Fragen eine 4-stufige Antwortskala enthalten, wobei drei Kategorien Zustimmung ausdrücken und nur eine Kategorie Ablehnung ausdrückt. Durch diese Ungleichverteilung könnten Schulleiter/innen eher dazu tendieren Rahmenbedingungen als belastend einzustufen. Des Weiteren wurden Aspekte wie die Fähigkeiten des Lehrkörpers nur mit einem Item erfasst. Überhaupt liegen für den Belastungsfaktor „Lehrer/innen“ nur zwei Items vor, weshalb die Faktorreliabilität eingeschränkt ist. Mit Blick auf die erforschten Effekte muss auf den Querschnittscharakter verwiesen werden: Die beobachteten Effekte könnten sich durchaus aus Wechselwirkungen zusammensetzen. Hier sind Längsschnittuntersuchungen nötig. Trotz dieser Einschränkungen besitzt die Studie Neuigkeitswert: Erstmals wurden Kontexteffekte aus Sicht der Schulleiter/ innen empirisch auf Basis einer Vollerhebung modelliert, sodass Zusammenhänge 
zwischen den aus Schulleitersicht wahrgenommenen Belastungsfaktoren am Schulstandort und dem Lehrerverhalten sowie dem Lernerfolg der Schüler/innen bestätigt werden konnten. Darüber hinaus konnte gezeigt werden, dass der Sozialindex einer Schule nicht nur, wie gemeinhin bekannt, im Zusammenhang mit der Schülerleistung steht, sondern auch mit der schülerzentrierten Unterrichtsgestaltung.

Open access funding provided by Johannes Kepler University Linz.

Open Access Dieser Artikel wird unter der Creative Commons Namensnennung 4.0 International Lizenz (http://creativecommons.org/licenses/by/4.0/deed.de) veröffentlicht, welche die Nutzung, Vervielfältigung, Bearbeitung, Verbreitung und Wiedergabe in jeglichem Medium und Format erlaubt, sofern Sie den/die ursprünglichen Autor(en) und die Quelle ordnungsgemäß nennen, einen Link zur Creative Commons Lizenz beifügen und angeben, ob Änderungen vorgenommen wurden.

\section{Literatur}

Agirdag, O., van Avermaet, P., \& van Houtte, M. (2013). School segregation and math achievement: a mixed-method study on the role of self-fulfilling prophecies. Teachers College Record, 115(3), 1-50.

Alborz, A., Pearson, D., Farrell, P., \& Howes, A. (2009). The impact of adult support staff on pupils and mainstream schools. Technical Report. University of London: Institute of Education.

Bacher, J., Altrichter, H., \& Nagy, G. (2010). Ausgleich unterschiedlicher Rahmenbedingungen schulischer Arbeit durch eine indexbasierte Mittelverteilung. Erziehung und Unterricht, 160(3-4), 384-400.

Baumert, J., \& Kunter, M. (2006). Stichwort: Professionelle Kompetenz von Lehrkräften. Zeitschrift für Erziehungswissenschaft, 9(4), 469-520.

Baumert, J., \& Kunter, M. (2011). Das mathematikspezifische Wissen von Lehrkräften, kognitive Aktivierung im Unterricht und Lernfortschritte von Schülerinnen und Schülern. In M. Kunter, J. Baumert, W. Blum, U. Klusmann, S. Krauss \& M. Neubrand (Hrsg.), Professionelle Kompetenz von Lehrkräften. Ergebnisse des Forschungsprogramms COACTIV (S. 163-192). Münster: Waxmann.

Baumert, J., Kunter, M., Blum, W., Brunner, M., Voss, T., Jordan, A., Klusmann, U., Krauss, S., Neubrand, M., \& Tsai, Y.-M. (2010). Teachers' mathematical knowledge, cognitive activation in the classroom, and student progress. American Educational Research Journal, 47(1), 133-180.

Biedermann, H., Weber, C., Herzog-Punzenberger, B., \& Nagel, A. (2015). Auf die Mitschüler/innen kommt es an? Schulische Segregation - Effekte der Schul- und Klassenzusammensetzung in der Primarstufe und der Sekundarstufe I. In M. Bruneforth, F. Eder, K. Krainer, C. Schreiner, A. Seel \& C. Spiel (Hrsg.), Nationaler Bildungsbericht Österreich (Bd. 2, S. 133-173). Graz: Leykam.

BIFIE (2012a). Schulleiterfragebogen. Überprüfung der Bildungsstandards Mathematik 8. Schulstufe. https://www.bifie.at/system/files/dl/BIST-UE_M8_SLFB.pdf. Zugegriffen: 18. Juli 2016.

BIFIE (2012b). Schülerfragebogen. Standardüberprüfung 8. Schulstufe. https://www.bifie.at/system/files/ dl/BIST-UE_2012_SFB_20120227.pdf. Zugegriffen: 18. Juli 2016.

Boekaerts, M. (1999). Self-regulated learning: Where we are today. International Journal of Educational Research, 31(6), 445-475.

Bonsen, M. (2010). Distributed leadership in der Schule. Erziehung und Unterricht, 160(7-8), 619-627.

Brauckmann, S., \& Schwarz, A. (2014). Autonomous leadership and a centralised school system - an odd couple? Empirical insights from Cyprus. International Journal of Educational Management, 28(7), 823-841.

Brauckmann, S., \& Schwarz, A. (2015). No time to manage? The trade-off between relevant tasks and actual priorities of school leaders in Germany. International Journal of Educational Management, 29(6), 749-765.

Browne, M. W., \& Cudeck, R. (1993). Alternative ways of assessing model fit. In K. A. Bollen \& J. S. Long (Hrsg.), Testing structural equation models (S. 136-162). Beverly Hills, CA: Sage.

Bruneforth, M., Weber, C., \& Bacher, J. (2012). Chancengleichheit und garantiertes Bildungsminimum in Österreich. In B. Herzog-Punzenberger (Hrsg.), Nationaler Bildungsbericht Österreich Fokussierte Analysen bildungspolitischer Schwerpunktthemen, (Bd. 2, S. 189-228). Graz: Leykam.

Darling-Hammond, L. (2000). Teacher quality and student achievement: a review of state policy evidence. Education Policy Analysis Archives, 8(1). http://epaa.asu.edu/ojs/article/view/392/515. Zugegriffen: 19. Juli 2016. 
Deci, E.L., \& Ryan, R.M. (1993). Die Selbstbestimmungstheorie der Motivation und ihre Bedeutung für die Pädagogik. Zeitschrift für Pädagogik, 39(2), 223-238.

Decristan, J., Fauth, B., Rieser, S., Büttner, G., \& Klieme, E. (2016). Kognitive Aktivierung in leistungsheterogenen Klassen. Abstractband zur GEBF 2016, Berlin. http://www.gebf2016.de/aktuelles/ Symposien_Abstracts_240216.pdf. Zugegriffen: 19. Juli 2016.

Ditton, H. (2000). Qualitätskontrolle und Qualitätssicherung in Schule und Unterricht. Ein Überblick zum Stand der empirischen Forschung. Zeitschrift für Pädagogik, Beiheft 41, 73-92.

Fauth, B., Decristan, J., Topalak, C., Rieser, S., Klieme, E., \& Büttner, G. (2016). Differentielle Effekte des Unterrichtsklimas in der Grundschule. Abstractband zur GEBF 2016, Berlin. http://www.gebf2016. de/aktuelles/Symposien_Abstracts_240216.pdf. Zugegriffen: 1. April 2016.

Helm, C., \& Altrichter, H. (2012). Wie können Jugendliche für die Schule begeistert werden? Evaluation eines neuen Handelsschulmodells mit Blick auf dessen Retentionskraft. In G. Niedermair (Hrsg.), Evaluation als Herausforderung der Berufsbildung und Personalentwicklung (S. 285-305). Linz: Trauner.

Helm, C., Kemethofer, D., Altrichter, H., \& Weber, C. (2015). Effekte der NMS-Konzeptmerkmale auf die fachlichen Schülerleistungen. In F. Eder, H. Altrichter, F. Hofmann \& C. Weber (Hrsg.), Bericht über die Evaluierung der „Neuen Mittelschule“ (NMS). Befunde aus den Anfangskohorten (S. 285-302). Graz: Leykam.

Helmke, A. (2009). Unterrichtsqualität und Lehrerprofessionalität. Diagnose, Evaluation und Verbesserung des Unterrichts. Seelze: Klett-Kallmeyer.

Helmke, A., \& Weinert, F. E. (1997). Bedingungsfaktoren schulischer Leistungen. In F. E. Weinert (Hrsg.), Psychologie des Unterrichts und der Schule (S. 71-176). Göttingen: Hogrefe.

Hill, C.H., Rowan, B., \& Ball, D.L. (2005). Effects of teachers' mathematical knowledge for teaching on student achievement. American Educational Research Journal, 42(2), 371-406.

Hu, L., \& Bentler, P. M. (1999). Cutoff criteria for fit indexes in covariance structure analysis: Conventional criteria versus new alternatives. Structural Equation Modeling: A Multidisciplinary Journal, 6(1), $1-55$.

Huber, S.G., \& Niederhuber, A. S. (2004). Schulleitung aus der Sicht von Lehrkräften. Pädagogik, 7(8), 44-47.

Kemethofer, D., Zuber, J., Helm, C., Demski, D., \& Rieß, C. (2015). Effekte von Schulentwicklungsmaßnahmen auf Schüler/innenleistungen im Fach Mathematik. SWS-Rundschau, 55(1), 26-47.

Klieme, E., \& Kuger, S. (2016). Umgang mit Heterogenität an Schulen des Sekundarbereichs im internationalen Vergleich. Abstractband zur GEBF 2016, Berlin. http:/www.gebf2016.de/aktuelles/ Symposien_Abstracts_240216.pdf. Zugegriffen: 1. April 2016.

König, J., Rothland, M., Darge, K., Lünnemann, M., \& Tachtsoglou, S. (2013). Erfassung und Struktur berufswahlrelevanter Faktoren für die Lehrerausbildung und den Lehrerberuf in Deutschland, Österreich und der Schweiz. Zeitschrift für Erziehungswissenschaft, 16(3), 553-577.

Kuhn, J.-T., \& Kiefer, T. (2013). Optimal Test Assembly in Practice. The Design of the Austrian Educational Standards Assessment in Mathematics. Zeitschrift für Psychologie, 221(3), 190-200.

Kunter, M. (2011). Forschung zur Lehrermotivation. In E. Terhart, H. Bennewitz \& M. Rothland (Hrsg.), Handbuch der Forschung zum Lehrerberuf (S. 527-539). Münster: Waxmann.

Kunter, M., Klusmann, U., Baumert, J., Richter, D., Voss, T., \& Hachfeld, A. (2013). Professional competence of teachers: Effects on instructional quality and student development. Journal of Educational Psychology, 105(3), 805-820.

Little, T. (2013). Longitudinal structural equation modeling. New York: The Guilford Press.

Liu, H., van Damme, J., Gielen, S., \& van den Noortgate, W. (2015). School processes mediate school compositional effects: model specification and estimation. British Educational Research Journal, 41(3), 423-447.

Lüdtke, O., Robitzsch, A., Trautwein, U., \& Kunter, M. (2009). Assessing the impact of learning environments: How to use student ratings in multilevel modelling. Contemporary Educational Psychology, 34, 120-131.

Marsh, H.W., Lüdtke, O., Robitzsch, A., Trautwein, U., Asparouhov, T., Muthén, B., \& Nagengast, B. (2009). Doubly-latent models of school contextual effects: Integrating multilevel and structural equation approaches to control measurement and sampling errors. Multivariate Behavioral Research, 44(6), 764-802.

Moosbrugger, R., Helm, C., Kemethofer, D., Bröderbauer, S., \& Luthe, S. (2016). Standortspezifische Belastungsfaktoren des schulischen Lernens - eine qualitative Analyse von Schulleiteraussagen. Die Deutsche Schule, 108(4), 412-430. 
Müller, F. H., Andreitz, I., \& Palekcic, M. (2008). Lehrermotivation - Ein vernachlässigtes Thema in der empirischen Forschung. Odgojne znanosti, 10(1), 39-60.

Muthén, L. K., \& Muthén, B. O. (1998-2014). Mplus user's guide (7. Aufl.). Los Angeles, CA: Muthén \& Muthén.

OECD (2014). Teaching in focus. https://www.oecd.org/edu/school/TIF5.pdf. Zugegriffen: 1. April 2016.

Opdenakker, M.-C., \& van Damme, J. (2001). Relationship between school composition and characteristics of school process and their effect on mathematics achievement. British Educational Research Journal, 27(4), 407-432.

Palardy, G. J. (2008). Differential school effects among low, middle, and high social class composition schools: a multiple group, multilevel latent growth curve analysis. School Effectiveness and School Improvement, 19(1), 21-49.

Pelletier, L. G., Séguin-Lévesque, C., \& Legault, L. (2002). Pressure from above and pressure from below as determinants of teachers' motivation and teaching behaviors. Journal of Educational Psychology, 94(1), 186-196.

Pietsch, M. (2014). Was wissen wir über wirksame Schulleitungen? Eine Zusammenschau und praxisorientierte Einordnung von Best-Evidence-Forschungsbefunden der letzten 10 Jahre. Journal für Schulentwicklung, 18(2), 15-23.

Rjosk, C., Richter, D., Hochweber, J., Lüdtke, O., Klieme, E., \& Stanat, P. (2014). Socioeconomic and language minority classroom composition and individual reading achievement: The mediating role of instructional quality. Learning and Instruction, 32, 63-72.

Roth, G., Assor, A., Kanat-Maymon, Y., \& Kaplan, H. (2007). Autonomous motivation for teaching: How self-determined teaching may lead to self-determined learning. Journal of Educational Psychology, 99(4), 761-774.

Rubin, D. B. (1987). Multiple imputation for nonresponse in surveys. New York: John Wiley \& Sons.

Scharenberg, K. (2012). Leistungsheterogenität und Kompetenzentwicklung. Zur Relevanz klassenbezogener Kompositionsmerkmale im Rahmen der KESS-Studie. Münster: Waxmann.

Scharenberg, K., Rollett, W., \& Bos, W. (2015). Stehen Merkmale der Klassenkomposition in einem Zusammenhang mit dem Beanspruchungsempfinden von Grundschullehrkräften? Unterrichtswissenschaft, 43(2), 101-119.

Schmich, J. (2010). Ressourcen an Schulen und Unterrichtsbeeinträchtigungen - wunder Punkt in Österreich? In J. Schmich \& C. Schreiner (Hrsg.), TALIS 2008: Schule als Lernumfeld und Arbeitsplatz. Vertiefende Analysen aus österreichischer Perspektive (S. 127-142). Graz: Leykam.

Schreiner, C., \& Breit, S. (2012). Standardüberprüfung 2012 Mathematik, 8. Schulstufe. Bundesergebnisbericht. https://www.bifie.at/system/files/d1/01_BiSt-UE_M8_2012_Bundesergebnisbericht.pdf. Zugegriffen: 24. März 2016.

Shulman, L.S. (1986). Those who understand: Knowledge growth in teaching. Educational Researcher, 15(2), 4-14.

Shulman, L.S. (1987). Knowledge and teaching: foundations of the new reform. Harvard Educational Review, 57, 1-22.

Warwas, J. (2011). Berufliches Selbstverständnis, Beanspruchung und Bewältigung in der Schulleitung. Wiesbaden: Springer VS.

Warwas, J. (2015). Principals' leadership behaviour: values-based, contingent or both? Journal of Educational Administration, 53(3), 310-334.

Yuan, K.H., \& Bentler, P.M. (2000). Three likelihood-based methods for mean and covariance structure analysis with non-normal missing data. Sociological Methodology, 30(1), 165-200. 